\title{
The Class of Absolute Decomposable Inequality Measures
}

\author{
Kristof Bosmans $^{*}$ and Frank A. Cowell ${ }^{\dagger}$
}

DARP 99

February 2009
The Toyota Centre

Suntory and Toyota International

Centres for Economics and Related

Disciplines

London School of Economics

Houghton Street

London WC2A 2A

(+44 020) 7955 6674)

\footnotetext{
* Maastricht University. Correspondence: Kristof Bosmans, Department of Economics, Maastricht University, Tongersestraat 53, 6211 LM Maastricht, The Netherlands.

e-mail: k.bosmans@algec.unimaas.nl

${ }^{\dagger}$ London School of Economics
} 


\begin{abstract}
We provide a parsimonious axiomatisation of the complete class of absolute nequality indices. Our approach uses only a weak form of decomposability and does not require a priori that the measures be differentiable.
\end{abstract}

Keywords: inequality measures, decomposability, translation invariance

JEL codes: D63, H20, H21 


\section{Distributional Analysis Research Programme}

The Distributional Analysis Research Programme was established in 1993 with funding from the Economic and Social Research Council. It is located within the Suntory and Toyota International Centres for Economics and Related Disciplines (STICERD) at the London School of Economics and Political Science. The programme is directed by Frank Cowell. The Discussion Paper series is available free of charge. To subscribe to the DARP paper series, or for further information on the work of the Programme, please contact our Research Secretary, Leila Alberici on:

Telephone: UK+20 79556674

Fax: $\quad$ UK+20 79556951

Email:_l.alberici@lse.ac.uk

Web site: $\quad$ http://sticerd.lse.ac.uk/DARP

(c) Authors: Kristof Bosmans and Frank A. Cowell. All rights reserved. Short sections of text, not to exceed two paragraphs, may be quoted without explicit permission provided that full credit, including (๑) notice, is given to the source. 


\section{Introduction}

Absolute inequality indices have the property that, for any income distribution, if any given number of dollars is added to (or subtracted from) every income, inequality remains unchanged. This means that such indices have the property that they are defined for negative incomes: this is particularly important in empirical applications where one is interested in applying inequality indices to the distribution of wealth (net worth may often be substantially negative) or to specific components of income (for example business income). Decomposability of inequality indices requires that, for an arbitrary subgroup of the population, if inequality in the subgroup increases then, ceteris paribus, inequality overall increases. Previous descriptions and characterizations of the class of indices that has these properties have invoked additional assumptions about structure - see section 4 below. Our approach (in sections 2 and 3) is deliberately minimalist in that it uses just the axioms required to define (a) an inequality measure (b) the "absolutist" property and (c) the population structure.

\section{Setting}

The income of individual $i$ is a real number $x_{i}$, and the income distribution for a population of $n$ individuals is a vector $\mathbf{x}:=\left(x_{1}, x_{2}, \ldots, x_{n}\right)$ in $\mathbb{R}^{n}$. The set of income distributions for $m$ or more individuals is $X_{m}:=\bigcup_{n=m}^{\infty} \mathbb{R}^{n}$. For an income distribution $\mathbf{x}$, we write $n(\mathbf{x})$ for the dimension and $\mu(\mathbf{x})$ for the mean. A vector of dimension $n$ of which all components are equal to 1 is denoted by $\mathbf{1}_{n}$.

An inequality measure is a continuous function $I: X_{1} \rightarrow \mathbb{R}$ with the property that $I(\mathbf{x})=0$ if $\mathbf{x}$ is an equal income distribution. The higher the value of the function $I$, the higher income inequality. To give meaning to $I$ it is standard to assume the following two properties:

Anonymity. For every $\mathbf{x} \in X_{1}, I(\mathbf{x})=I\left(\mathbf{x}^{\prime}\right)$ if $\mathbf{x}^{\prime}$ is obtained from $\mathbf{x}$ by rearrangement of components.

Transfer principle. For every $\mathrm{x} \in X_{2}$ and any positive real number $\delta$, we have that if $x_{i}<$ $x_{i}+\delta \leq x_{j}-\delta<x_{j}$, then $I\left(x_{1}, \ldots, x_{i}, \ldots, x_{j}, \ldots, x_{n}\right)>I\left(x_{1}, \ldots, x_{i}+\delta, \ldots, x_{j}-\delta, \ldots, x_{n}\right)$.

The essential property for an abolute inequality measure is this:

Translation invariance. For every $\mathbf{x} \in X_{1}$ and any real number $\delta$,

$$
I(\mathbf{x})=I\left(\mathbf{x}+\delta \mathbf{1}_{n}\right)
$$

Finally, the following two axioms describe the relationship between population structure and inequality:

Decomposability. There exists a function $A$ such that, for all $\mathbf{x}, \mathbf{y} \in X_{2}$,

$$
I(\mathbf{x}, \mathbf{y})=A(I(\mathbf{x}), I(\mathbf{y}), \mu(\mathbf{x}), \mu(\mathbf{y}), n(\mathbf{x}), n(\mathbf{y})),
$$

where $A$ is continuous and strictly increasing in its first two arguments.

Replication invariance. For every $\mathbf{x} \in X_{1}, I(\mathbf{x}, \mathbf{x})=I(\mathbf{x})$. 


\section{Result}

Our result characterizes the class of inequality measures satisfying the five properties listed in section 2 .

Theorem. An inequality measure I satisfies anonymity, the transfer principle, replication invariance, decomposability and translation invariance if and only if there exists a real number $c$ and a continuous and strictly increasing function $f: \mathbb{R} \rightarrow \mathbb{R}$, with $f(0)=0$, such that, for all $\mathbf{x} \in X_{1}$,

$$
f(I(\mathbf{x}))= \begin{cases}\frac{1}{n(\mathbf{x})} \sum_{i=1}^{n(\mathbf{x})}\left\{e^{c\left[x_{i}-\mu(\mathbf{x})\right]}-1\right\} & \text { if } c \neq 0, \\ \frac{1}{n(\mathbf{x})} \sum_{i=1}^{n(\mathbf{x})}\left[x_{i}-\mu(\mathbf{x})\right]^{2} & \text { if } c=0 .\end{cases}
$$

Proof. The inequality measures given in (2) satisfy anonymity, the transfer principle, replication invariance, decomposability and translation invariance. We focus on the reverse implication.

If $I$ satisfies anonymity, the transfer principle, replication invariance and decomposability, then (Shorrocks, 1984, Theorem 4) there exist functions $F$ and $\phi$ such that

$$
F(I(\mathbf{x}), \mu(\mathbf{x}), n(\mathbf{x}))=\sum_{i=1}^{n(\mathbf{x})}\left[\phi\left(x_{i}\right)-\phi(\mu(\mathbf{x}))\right],
$$

where $\phi$ is continuous and strictly convex, and where $F$ is continuous in $I$ and $\mu$, strictly increasing in $I$, additive in $n$, with $F(0, \mu, n)=0$. Choose any $\mathbf{x}_{1}$ and $\mathbf{x}_{2}$ in $X_{2}$, and write $\mathbf{x}_{12}:=\left(\mathbf{x}_{1}, \mathbf{x}_{2}\right), I_{k}:=I\left(\mathbf{x}_{k}\right), n_{k}:=n\left(\mathbf{x}_{k}\right)$ and $\mu_{k}:=\mu\left(\mathbf{x}_{k}\right)$. We may write

$$
I\left(\mathbf{x}_{12}\right)=H\left(F\left(I_{1}, \mu_{1}, n_{1}\right)+F\left(I_{2}, \mu_{2}, n_{2}\right), \mu_{1}, \mu_{2}, n_{1}, n_{2}\right)
$$

where $H$ is a function implicitly defined by

$$
\left.F\left(H\left(z, \mu_{1}, \mu_{2}, n_{1}, n_{2}\right)\right), \mu_{1}, \mu_{2}, n_{1}, n_{2}\right)=z+n_{1} \phi\left(\mu_{1}\right)+n_{2} \phi\left(\mu_{2}\right)-n_{12} \phi\left(\mu_{12}\right) .
$$

Define $\lambda:=e^{\delta}$ and $\theta_{k}:=e^{\mu_{k}}$. Then $\lambda \theta_{k}=e^{\mu_{k}+\delta}$, and (4) and (1) imply

$$
\begin{aligned}
& H\left(F\left(I_{1}, \ln \left(\theta_{1}\right), n_{1}\right)+F\left(I_{2}, \ln \left(\theta_{2}\right), n_{2}\right), \ln \left(\theta_{1}\right), \ln \left(\theta_{2}\right), n_{1}, n_{2}\right) \\
& \quad=H\left(F\left(I_{1}, \ln \left(\lambda \theta_{1}\right), n_{1}\right)+F\left(I_{2}, \ln \left(\lambda \theta_{2}\right)\right), \ln \left(\lambda \theta_{1}\right), \ln \left(\lambda \theta_{2}\right), n_{1}, n_{2}\right),
\end{aligned}
$$

or, with appropriate definitions of $G$ from $F$ and of $J$ from $H$,

$$
\begin{aligned}
& J\left(G\left(I_{1}, \theta_{1}, n_{1}\right)+G\left(I_{2}, \theta_{2}, n_{2}\right), \theta_{1}, \theta_{2}, n_{1}, n_{2}\right) \\
& \quad=J\left(G\left(I_{1}, \lambda \theta_{1}, n_{1}\right)+G\left(I_{2}, \lambda \theta_{2}, n_{2}\right), \lambda \theta_{1}, \lambda \theta_{2}, n_{1}, n_{2}\right) .
\end{aligned}
$$

From Lemma 1 in Shorrocks (1984):

$$
\begin{aligned}
& G\left(I_{1}, \lambda \theta_{1}, n_{1}\right)=c G\left(I_{1}, \theta_{1}, n_{1}\right) \\
& G\left(I_{2}, \lambda \theta_{2}, n_{2}\right)=c G\left(I_{2}, \theta_{2}, n_{2}\right)
\end{aligned}
$$


from which it follows that

$$
c=\frac{G(I, \lambda \theta, n)}{G(I, \theta, n)}=c(\lambda)
$$

independent of $I, \mu$ and $n$. Therefore

$$
\begin{aligned}
G(I, \lambda \theta, n) & =c(\lambda) G(I, \theta, n) \\
& =c(\lambda) c(\theta) G(I, 1, n) \\
& =c(\lambda \theta) G(I, 1, n) .
\end{aligned}
$$

Because $c(\lambda \theta)=c(\lambda) c(\theta)$ for all positive $\lambda$ and $\theta$, we have $c(\theta)=\theta^{c}$ (Aczél, 2006, Theorem 4 , p. 144). Consequently

$$
\begin{aligned}
G(I, \theta, n) & =\theta^{c} G(I, 1, n), \\
F(I, \mu, n) & =e^{\mu c} F(I, 0, n),
\end{aligned}
$$

and (3) yields

$$
F(I(\mathbf{x}), 0, n)=e^{-\mu c} \sum_{i=1}^{n}\left[\phi\left(x_{i}\right)-\phi(\mu)\right] .
$$

In the case of two individuals,

$$
0=F\left(I\left(\mathbf{x}+\delta \mathbf{1}_{2}\right), \mu(\mathbf{x})+\delta, 2\right)-e^{c \delta} F(I(\mathbf{x}), \mu(\mathbf{x}), 2),
$$

and writing $\mathbf{x}:=(u, v)$ this becomes

$$
0=\left[\phi(u+\delta)+\phi(v+\delta)-2 \phi\left(\frac{u+v}{2}+\delta\right)\right]-e^{c \delta}\left[\phi(u)+\phi(v)-2 \phi\left(\frac{u+v}{2}\right)\right] .
$$

If we define

$$
\psi(u, \delta):=\phi(u+\delta)-e^{c \delta} \phi(u)
$$

then (6) becomes

$$
0=\psi(u, \delta)+\psi(v, \delta)-2 \psi\left(\frac{u+v}{2}, \delta\right) .
$$

This is a Pexider equation with solution (Aczél, 2006, p. 142)

$$
\psi(u, \delta)=a(\delta) u+b(\delta)
$$

From (7),

$$
\begin{aligned}
\psi(u, \mu+\delta) & =\phi(u+\mu+\delta)-e^{c[\mu+\delta]} \phi(u) \\
& =\psi(u+\mu, \delta)+e^{c \delta} \phi(u+\mu)-e^{c[\mu+\delta]} \phi(u) \\
& =\psi(u+\mu, \delta)+e^{c \delta} \psi(u, \mu) .
\end{aligned}
$$

Substitute from (8) into (9) to get

$$
a(\mu+\delta) u+b(\mu+\delta)=a(\delta)(u+\mu)+b(\delta)+e^{c \delta}[a(\mu) u+b(\mu)],
$$


which implies

$$
\begin{aligned}
& a(\mu+\delta)=a(\delta)+e^{c \delta} a(\mu)=a(\mu)+e^{c \mu} a(\delta), \\
& b(\mu+\delta)=a(\delta) \mu+b(\delta)+e^{c \delta} b(\mu)=a(\mu) \delta+b(\mu)+e^{c \mu} b(\delta) .
\end{aligned}
$$

There are two cases to consider.

Case $1(c \neq 0)$. In this case $(10)$ gives

$$
\begin{aligned}
a(\delta)\left(e^{c \mu}-1\right) & =a(\mu)\left(e^{c \delta}-1\right), \\
a(\mu) & =\frac{a(\delta)}{e^{c \delta}-1}\left(e^{c \mu}-1\right),
\end{aligned}
$$

so that, for any given value of $\delta$ and all $\mu$,

$$
a(\mu)=\frac{k_{1}}{k_{2}}\left(e^{c \mu}-1\right),
$$

where $k_{1}:=a(\delta)$ and $k_{2}:=e^{c \delta}-1$ can be taken as constants (conditional on the chosen $\delta$ and the arbitrary value of $c$ ). So the only solution is

$$
a(\mu)=\alpha\left(e^{c \mu}-1\right) .
$$

Therefore, from (11)

$$
b(\mu+\delta)=\mu \alpha\left(e^{c \delta}-1\right)+b(\delta)+e^{c \delta} b(\mu)=\delta \alpha\left(e^{c \mu}-1\right)+b(\mu)+e^{c \mu} b(\delta),
$$

so that

$$
\begin{aligned}
\mu \alpha\left(e^{c \delta}-1\right)+b(\mu)\left(e^{c \delta}-1\right) & =\delta \alpha\left(e^{c \mu}-1\right)+b(\delta)\left(e^{c \mu}-1\right), \\
b(\mu)\left(e^{c \delta}-1\right) & =[\delta \alpha+b(\delta)]\left(e^{c \mu}-1\right)-\mu \alpha\left(e^{c \delta}-1\right), \\
b(\mu) & =\frac{\delta \alpha+b(\delta)}{e^{c \delta}-1}\left(e^{c \mu}-1\right)-\mu \alpha .
\end{aligned}
$$

Since this must be true for arbitrary $\delta$ and $c$, the solution is

$$
b(\mu)=\beta\left(e^{c \mu}-1\right)-\alpha \mu .
$$

Case $2(c=0)$. Here (10) becomes

$$
a(\mu+\delta)=a(\mu)+a(\delta)
$$

and the solution to this Cauchy equation is $a(\mu)=\kappa \mu$ (Aczél, 2006, Theorem 2, pp. 35-36). Therefore, from (11)

$$
b(\mu+\delta)=\kappa \mu \delta+b(\delta)+b(\mu),
$$

Using that $b(0)=0$ and letting $\delta=-\mu$ and $\kappa=2 \gamma$, we get

$$
b(\mu)+b(-\mu)=2 \gamma \mu^{2},
$$


to which the solution is

$$
b(\mu)=\gamma \mu^{2}+p(\mu),
$$

where $p(\mu):=\Pi(\mu,-\mu)$ with the property $p(\mu)=-p(-\mu)$ (Polyanin and Manzhirov, 1998). Plugging this into (13) gives

$$
p(\mu+\delta)=p(\mu)+p(\delta)
$$

to which the solution is $p(\mu)=\eta \mu$. Therefore

$$
b(\mu)=\gamma \mu^{2}+\eta \mu .
$$

From (8), we have $\psi(0, x)=b(x)$; so from (12) and (14) we find

$$
\psi(0, x)= \begin{cases}\beta\left(e^{c x}-1\right)-\alpha x & \text { if } c \neq 0, \\ \gamma x^{2}+\eta x & \text { if } c=0 .\end{cases}
$$

Note that, from (7), we have:

$$
\phi(x)=\psi(0, x)+e^{c x} \phi(0) .
$$

However the constant $\phi(0)$ is arbitrary. Setting $\phi(0)=0$ and substituting from (15) and (16) into (5) produces (2).

\section{Concluding remarks}

While it is well known that the resulting measures in (2) belong to the class of absolute decomposable measures (Chakravarty and Tyagarupananda, 1998, 2009), it has not previously been demonstrated that only these measures belong to the class: previous treatments have introduced a priori additional restrictions such as differentiability and have used a stronger decomposability property.

Letting $c=1$, we obtain the variance and, letting $c<0$, the family (2) is ordinally equivalent to the Kolm (1976) family of measures. As the parameter $c$ decreases, the corresponding measures are more sensitive to transfers between incomes at the bottom of the income distribution. Note that the Kolm measures are the only members of the class of absolute decomposable measures satisfying transfer sensitivity (Shorrocks and Foster, 1987), which is the requirement that a rich-to-poor transfer combined with a simultaneous poor-to-rich transfer at a higher income level (such that there is no effect on the variance) decreases inequality.

\section{References}

Aczél J., 2006. Lectures on Functional Equations and Their Applications. Mineola: Dover Publications, Inc.

Chakravarty S.R. and S. Tyagarupananda, 1998. The subgroup decomposable absolute indices of inequality. In: S.R. Chakravarty, D. Coondoo and R. Mukherjee (eds) Quantitative Economics: Theory and Practice, Essays in Honor of Professor N. Bhattacharya. New Delhi: Allied Publishers Limited, 247-257. 
Chakravarty S.R. and S. Tyagarupananda, 2009. The subgroup decomposable intermediate indices of inequality. Forthcoming in Spanish Economic Review.

Kolm S.-C., 1976. Unequal inequalities I. Journal of Economic Theory 12, 416-442.

Polyanin A.D. and A.V. Manzhirov, 1998. Handbook of Integral Equations: Exact Solutions. Moscow: Faktorial.

Shorrocks A.F., 1984. Inequality decomposition by population subgroups. Econometrica 52, 1369-1385.

Shorrocks A.F. and J.E. Foster, 1987. Transfer sensitive inequality measures. Review of Economic Studies 54, 485-497. 